\title{
Body fat prediction equations for skinfold and bioelectrical impedance analysis using dual-energy $x$-ray absorptiometry data as the criterion.
}

\author{
Jared Grove, You-jou Hung* \\ Department of Physical Therapy, Angelo State University, USA
}

\begin{abstract}
Background and purpose

Body composition evaluation during health screenings and physical examination is important for health professionals (including physical therapists) to categorize health risk and prescribe appropriate exercise interventions. In addition to measuring bone mineral density, dual energy x-ray absorptiometry (DXA) can provide precise measurement of body fat percentage with minimal radiation exposure. However, having access to DXA is very costly and other common body fat measurements are no very accurate. The purpose of this study was to develop body fat prediction equations for bioelectrical impedance analysis (BIA) and skinfold measurement, using DXA data as the criterion.
\end{abstract}

\section{Methods}

This was a within-group study with repeated measures. Sixty three college age students participated in the study. Subject's body fat percentage was examined with DXA, BIA, and the 3-site skinfold measurements.

\section{Results}

Body fat percentage measured with DXA (26.27\%) is significantly higher than those measured with skinfold $(17.64 \%)$ and BIA $(20.70 \%)$. The DXA criterion regression equations were created for skinfold and BIA: $\mathrm{DXA} \% \mathrm{BF}=4.65+0.43 * \mathrm{~S} 3 \mathrm{SF}$ (sum of 3 site skinfold in $\mathrm{mm}$ ); $\mathrm{DXA} \% \mathrm{BF}=3.79+1.09 * \mathrm{BIA} \% \mathrm{BF}$. The new regression equations were further validated using $75 \%-25 \%$ subject cross-validation.

\section{Discussion}

Body fat percentage varies greatly among different measurements. Adjustments are necessary to accurately predict body fat percentage when using skinfold or BIA techniques at a clinical setting. The limitation of the study is that it is unclear if the results can be generalized to subjects of a different age group and ethnicities.

\section{Implication for physiotherapy practice}

Obtaining an accurate body fat percentage measurement is important for health promotion and cardiovascular risk screening. Although skin-fold and BIA equipment are more readily available, their results underestimate the body fat percentage and may mislead clinicians on the wellbeing of the patient.

Keywords Dual energy x-ray absorptiometry (DXA), Bioelectrical impedance analysis (BIA), Body density.

Accepted on November 25, 2017

\section{Introduction}

With an estimated $34.9 \%$ adult obesity rate in the United States, body composition evaluation during health screenings and physical examinations is an important tool for physical therapists to categorize health risk and prescribe appropriate exercise interventions [1]. Body mass index (BMI) is commonly used to classify individuals into different health risk categories because it can be conveniently and quickly calculated. However, using BMI as an indicator of body composition could be inaccurate and may lead to bias in evaluating health conditions [2]. To calculate BMI, one cannot differentiate lean body mass from fat mass. As the result, an individual with a large muscle mass could be categorized at the same level with the same health risks as those with a large fat mass.

There are many techniques for body composition measurements. Hydrostatic weighing (underwater weighing) is a two-component (fat mass and fat free mass) model and it has long been used in a laboratory setting for vivo body composition measurement [3-5]. The test is difficult to conduct in most clinical and fitness settings. Moreover, subject/ examiner errors can often occur during hydrostatic weighting without extensive practice. Despite being considered as the gold standard by many researchers, the two-component model neglects the individual variation in the composition of fat free mass (e.g., water, lean muscle, bone). Data from hydrostatic weighting was significantly different from those acquired from 
Citation: Grove J, Hung YJ. Body fat prediction equations for skinfold and bioelectrical impedance analysis using dual-energy x-ray absorptiometry data as the criterion. J Phys Ther Sports Med 2017;1(1):5-11.

the more accurate four-component model technique which divides body weight into fat mass, total body water, bone mineral mass, and residual [4-6] .

Skinfold measurement and bioelectrical impedance analysis (BIA) are 2 common methods for clinicians to examine body composition and estimate body fat percentage. Many skinfold measurement techniques (such as Jackson and Pollock's 3-site and 7-site protocols) and equations were developed to estimate body fat percentage for various populations [7-9]. However, factors such as poor technique of an inexperienced examiner can contribute to measurement errors [10]. Moreover, it is also very crucial to choose a proper population specific formula to convert body density to body fat percentage. BIA is another widely used body composition technique which is based on the speed of electrical current conduction through different types of body tissue. BIA is a reliable, non-invasive, and rapidly performed body composition technique. It is suggested that the accuracy of BIA testing is similar to skinfold testing with a normal/similar hydration level when proper testing protocols are followed [11-12]. In addition, BIA machine is also inexpensive and portable, ideal for non-hospital settings such as fitness facilities. However, due to the differences in body water distribution, BIA testing may not be valid when testing obese individuals [13].

Dual energy x-ray absorptiometry (DXA) was approved by the Food and Drug Administration in 1988[14]. It provides precise measurement of bone mineral content and soft tissue composition [15-21]. Results from DXA also have a strong correlation to the data examined with magnetic resonance imaging, computed tomography, and multi-compartmental models [23-26,22]. Being recognized as the new gold standard for measuring body composition [27-3], DXA measures three components (fat mass, lean mass, and bone mass) in the full body without making assumptions about their densities [31]. In addition, DXA has a very low radiation exposure [32], and the test can be completed in 6-20 minutes with minimal subject/ operator errors [27]. Despite DXA's accuracy in measuring body composition, one major limitation of using DXA is the expense of the machine and the cost to have a DXA scan performed. In addition, some individuals may not fit appropriately on the scanning table and additional procedures will need to be performed to accommodate those individuals [33-34].

Some studies have attempted to create group specific (e.g., children, professional athletes, obese women) prediction equations for Skinfold or BIA measurements using DXA as the criterion [3,27,36-38]. However, it is not clear if body fat percentage measured with skinfold and BIA is similar to those measured with DXA in college students. The first purpose of the present study was to compare body fat percentage measured by skinfold, BIA, and DXA techniques in college students. The second purpose of the present study was to develop body fat prediction equations for BIA and skinfold measurement, using DXA data as the criterion.

\section{Methods}

\section{Participants}

Sixty-three college age students (28 males and 35 females, age 18-27 years old) from a local university volunteered for this study. Subjects who were obese (BMI $\geq 30)$ or underweight $(\mathrm{BMI}<18.5)$, based on the guidelines of American College of Sports Medicine [10], were excluded from the study. Subjects with obesity may not fit on the DXA testing platform, which can hamper the DXA testing results. In addition, females who were pregnant or had the possibility of being pregnant were excluded from the study to avoid any radiation exposure from the DXA machine. All subjects signed a written informed consent in accordance with the policies and procedures Angelo State University human subjects institutional review board (IRB). They were treated properly according to the provisions of the Helsinki criteria to conduct research involving human subjects.

\section{Procedure}

Subjects were asked to wear athletic clothing (i.e., shorts, tshirt, sports bra) without metal parts at the day of testing. They were also informed not to perform exercise within several hours prior to the measurement because of factors (e.g., dehydration, increased vascular perfusion, warming of muscle tissue, increased skin temperature, and sweating) due to exercise can impact the accuracy of the testing. At the beginning of the testing, self-reported activity levels were categorized into 3 levels: very active-individuals who exercised at an intensity of $>8$ metabolic equivalents (METs) at least 3 to 5 days per week for sessions of at least 30 minutes each, moderately active - individuals who exercised at an intensity of 4 to 7 METs at least 3 days a week for sessions of at least 30 minutes each, and not active - individuals who exercised at an intensity of $<3$ METs. Exercise intensity examples for each category were provided to the subjects. Activity examples for "very active" individuals include fast walking, jogging ( $>5 \mathrm{mi} / \mathrm{h})$, hiking at steep grades, running, basketball competition, bicycling, volleyball competition, tennis single, swimming competition, and soccer competition. Activity examples for "moderately active" individuals include brisk walking, badminton, basketball (shooting a round), dancing, golf, sailing, table tennis, volleyball (noncompetitive), and tennis double. Activity examples for "not active" individuals include slow walking, billiards, boating, croquet, darts, and fishing. Subjects' body weight and height were measured using the DETECTO physician scale (Webb City, MO). Height and weight measurements were taken without shoes to the nearest 0.5 pounds and the nearest 0.5 inch. Their BMI was calculated subsequently. To reduce variability among 3 body composition measurements (skinfold, BIA, DXA), they were conducted in series within an hour in the afternoons. All measurements were taken by the same highly trained examiner to ensure consistency. To examine the test/re-test reliability, 20 subjects (10 males and 10 females) were tested again 24 hours after the original testing. 
A Lange skinfold calliper (Beta Technology Inc. Santa Cruse CA) and the three-site skinfold formula was used to examine body composition and body fat percentage. Skinfolds from chest, abdomen, and thigh were examined for males, [8] and skinfolds from triceps, suprailiac, and thigh were examined for females. [9] Two skinfolds were measured at each site to the nearest $0.5 \mathrm{~mm}$. A third measurement will be performed if the first 2 measurements differed by more than 1-2 $\mathrm{mm}$. The average of the closest 2 measurements was used for further analysis. Body density was calculated for males and females with separate equations based on ACSM guidelines [10]. Body density was further converted to body fat percentage with different formulas based on sex, age, and ethnicity [10].

BIA was analysed with a bio impedance analyser (BIA): BIA 450 (Bio dynamics Corp. Shoreline WA). The subject was positioned in supine on a non-conductive surface in an anatomical position with limbs slightly abducted (to avoid skin contact) and palms down. Each subject's personal information was entered into the data set of the BIA 450 including sex, age, height (inches), and weight (pounds). Skin sites were cleaned with an alcohol prep pad and allowed to dry. Four surface electrodes were placed on the right side of the body: dorsal surface of right wrist between radial and ulnar styloid processes, dorsal surface of right hand at space between second and third metacarpophalangeal joints, anterior surface of right ankle between medial and lateral malleoli, and anterior surface of right foot at space between first and second metatarsophalangeal joints. The subjects were asked to remain still and breathe normally during the testing (for a few seconds).

DXA (Hologic QDR 4500 X-ray Bone Densitometer, Bedford MA) was also used for body composition analysis. The machine was calibrated at the beginning of each testing period using a spine core calibration block. The subject was asked to remove all metal and/or jewellery prior to scanning, and was positioned in supine on the scanning table in an anatomical position with limbs slightly abducted (to avoid contacting the torso) and palms down. The legs and feet were positioned in slight internal rotation and held lightly with an elastic band. The subjects were instructed to stay still during the scanning process (scan time of 6 minutes).

\section{Data analysis}

The IBM Statistical Package for Social Sciences (SPSS) version 21 was used for all statistical analysis. Intraclass correlation coefficient (ICC) was used to examine test-retest reliability of skinfold, BIA, and DXA testing. Analysis of variance (ANOVA) was used to compare body fat percentage measured with different techniques. Pearson correlation was used to examine the body fat percentage measured from the 3 testing techniques and BMI. Bland-Altman Plots were created to identify any systematic or proportional biases within the data sets [39]. Simple linear regression analysis was performed to create body fat prediction equations for BIA and skinfold measurement, using DXA data as the criterion. The new regression equations were further validated using 75\%-25\%subject cross-validation. Significance level (p value) was set at 0.05 for all comparisons.

\section{Results}

Most of the subjects participated in the present study are Caucasian (85.7\%) and self-identified as very active $(55.6 \%)$ (Table1). For test-retest reliability, intraclass correlation coefficient (ICC) was high for skinfold $(r=.994)$, BIA $(r=.990)$, and DXA $(r=.997)$. Body fat percentage was significantly different $(\mathrm{p}<.01)$ among the 3 different body composition testing methods (skinfold: $17.64 \%$, BIA: $20.70 \%$, and DXA: $26.27 \%$ ) and between genders (male: $16.43 \%$, female: $25.62 \%$ ) (Figure1). Body fat percentage measured from DXA was significantly $(\mathrm{p}<.01)$ correlated with skinfold $(\mathrm{r}=.895)$, and BIA $(r=.875)$. However, none of the body fat percentage measurement was significantly correlated with BMI $(\mathrm{r}<.205)$.

Bland-Altman plots show both BIA and skinfold measurements underestimated body fat percentage compared to DXA, with a greater discrepancy between skinfold and DXA. The DXA criterion regression equations were created for skinfold and BIA: DXA $\% \mathrm{BF}=4.65+0.43 * \mathrm{~S} 3 \mathrm{SF}$ ( sum of 3 site skinfold in mm); DXA\%BF=3.79+1.09* BIA\%BF (Figure 2). Considering potential gender difference, separate regression analysis was performed for male and female subjects. For male subjects, DXA body fat percentage could be predicted from skinfold (DXA\%BF=5.54+0.38* S3SF) or BIA (DXA $\% \mathrm{BF}=5.95+0.94 * \mathrm{BIA} \% \mathrm{BF}$ ) (Figure 3). For female subjects, DXA body fat percentage could be predicted from skinfold $(\mathrm{DXA} \% \mathrm{BF}=9.15+0.37 * \mathrm{~S} 3 \mathrm{SF})$ or $\mathrm{BIA}(\mathrm{DXA} \% \mathrm{BF}=2.81+1.13$ * BIA\%BF) (Fig. 4). Due to the sample size, cross-validation for the prediction equations was only performed for all 63 subjects combining males and females. Regression equations were compared between $25 \%$ and $75 \%$ of the total participants. For skinfold to DXA prediction, cross-validation returned $(\mathrm{r}=$. $886)$ for $25 \%$ of the sample, which is similar $(r=.874)$ to the other $75 \%$ of the sample. For BIA prediction, cross-validation returned $(\mathrm{r}=.889)$ for $25 \%$ of the sample, which is similar $(\mathrm{r}=$. 875 ) to the other $75 \%$ of the sample.

Table 1. Descriptive statistics for the subjects $(N=63)$.

\begin{tabular}{ll}
\hline Variable & Mean \pm s.d. \\
\hline Age & $21.86 \pm 2.20$ \\
\hline Height (in.) & $67.51 \pm 4.25$ \\
\hline Weight (lbs.) & $153.71 \pm 28.08$ \\
\hline
\end{tabular}


Citation: Grove J, Hung YJ. Body fat prediction equations for skinfold and bioelectrical impedance analysis using dual-energy x-ray absorptiometry data as the criterion. J Phys Ther Sports Med 2017;1(1):5-11.

\begin{tabular}{lll}
\hline BMI & $23.55 \pm 2.55$ & \\
\hline Body fat & $17.64 \pm 6.69$ & \\
\hline Skinfold & $20.70 \pm 5.68$ & $26.27 \pm 7.04$ \\
\hline BIA & & $7(85.7 \%)$ \\
\hline DXA & $7(11.1 \%)$ \\
\hline Ethnicity & $1(1.6 \%)$ \\
\hline Caucasian & $1(1.6 \%)$ \\
\hline Hispanic & & $35(55.6 \%)$ \\
\hline African American & & $20(31.7 \%)$ \\
\hline Indian & & $8(12.7 \%)$ \\
\hline Fitness level & & \\
\hline Very Active & & \\
\hline Moderately Active & & \\
\hline Not Active & & \\
\hline
\end{tabular}

BMI: body mass index; BIA: bioelectrical impedance analysis;

DXA: dual-energy x-ray absorptiometry.

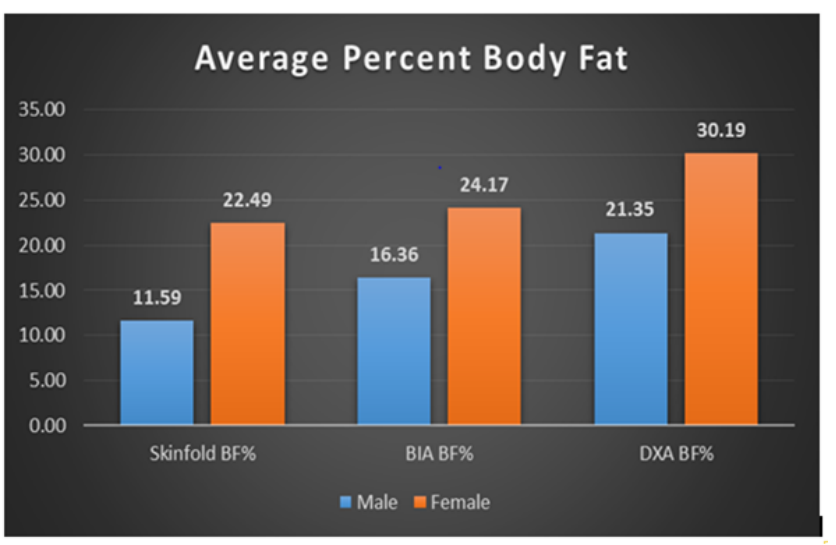

Figure 1. Mean body fat percentage measured with skinfold, BIA, and DXA (BIA: bioelectrical impedance analysis; DXA: dual-energy $x$-ray absorptiometry).
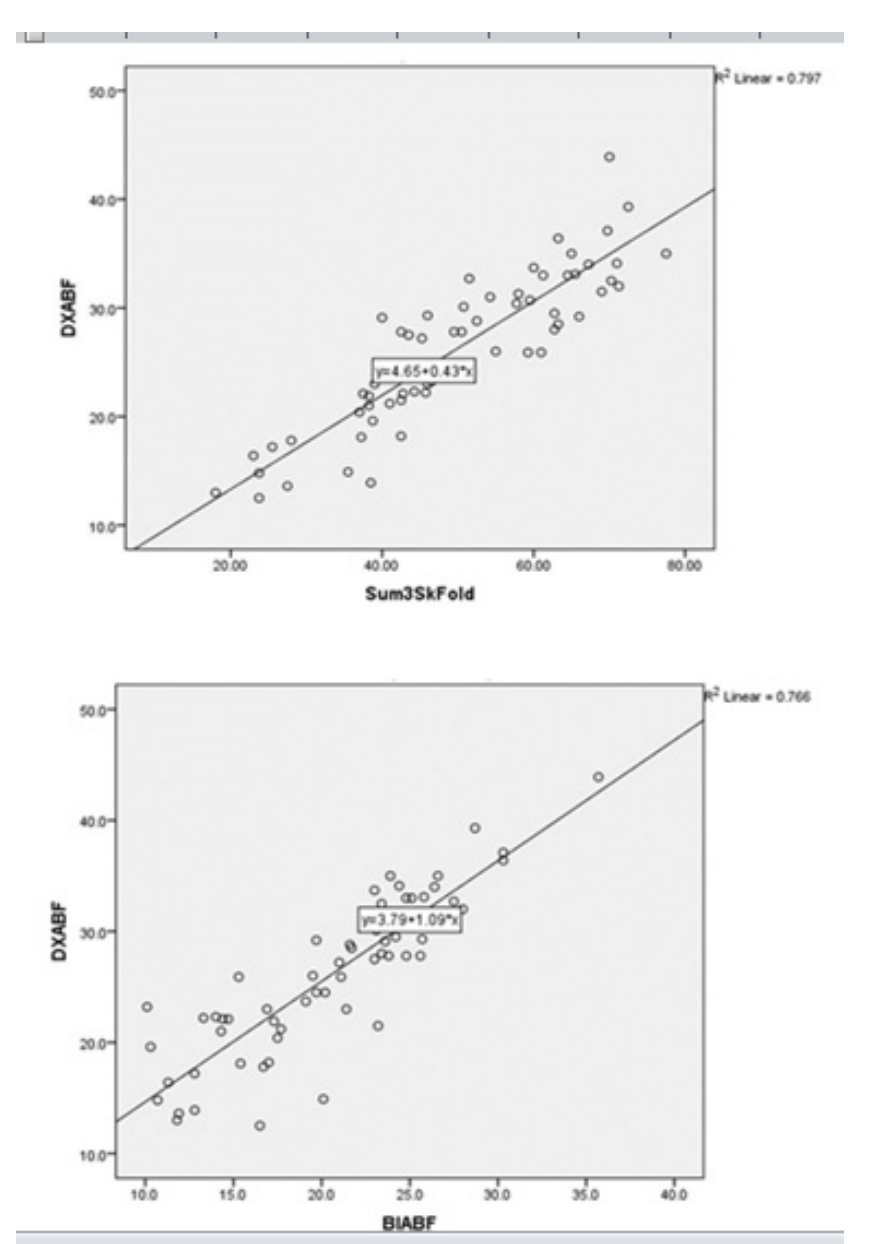

Figure 2. Simple linear regression using sum of the three skinfolds (2a) and BIA (2b) to predict DXA body fat percentage (DXABF: body fat percentage measured by dual-energy $x$-ray absorptiometry; Sum3SkFold: sum of the three skinfolds; BIABF: body fat percentage measured by bioelectrical impedance analysis). 


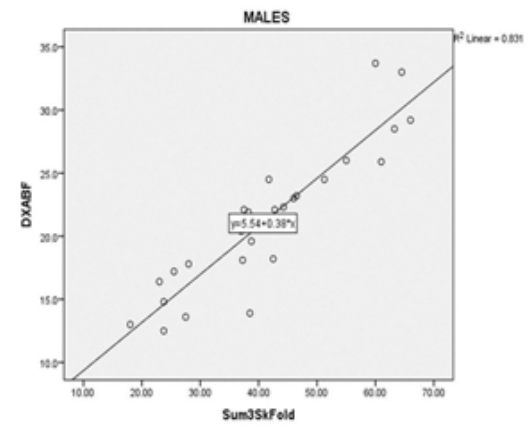

Figure 3A. Simple linear regression using sum of the three skinfolds.

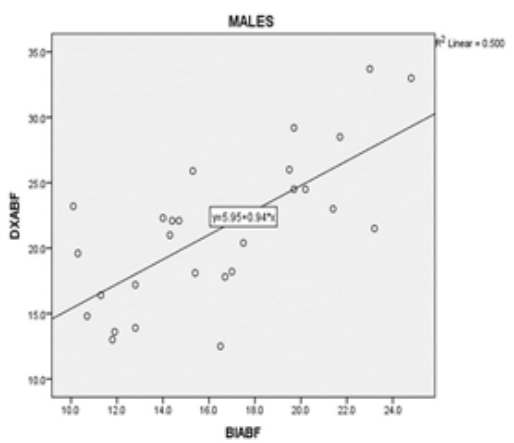

Figure 3B. Simple linear regression using sum of the BIA.

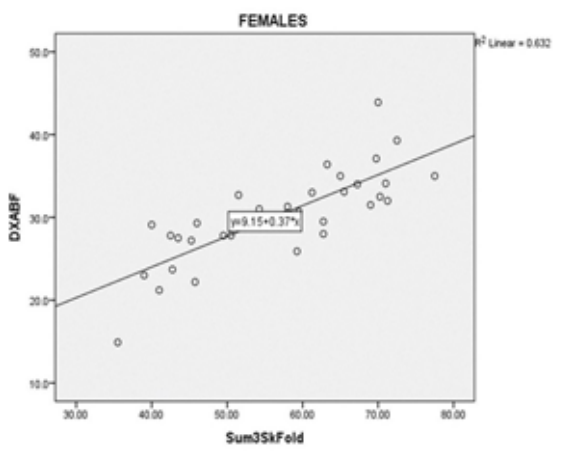

Figure 3C. To predict DXA body fat percentage for males $(N=28)$ using sum of the three skinfolds and BIA.

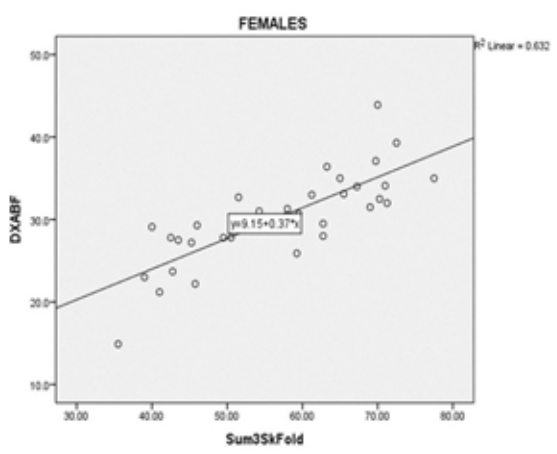

Figure 3D. To predict DXA body fat percentage for females $(N=35)$ using sum of the three skinfolds and BIA. (DXABF: body fat percentage measured by dual-energy x-ray absorptiometry; Sum 3SkFold: sum of the three skinfolds; BIABF: body fat percentage measured by bioelectrical impedance analysis).

\section{Discussion}

The purpose of this study was to compare body fat percentage examined with BIA and 3-sites skinfold analysis to the results examined with DXA in college students with a normal BMI. Results indicate that both skinfold and BIA body composition measurement significantly underestimate body fat percentage for college age students. The finding is consistent with prior studies that examined other populations such as 18-62 year old males and 18-55 year old females $[3,27]$. However, the data for the current study can better represent a specific population: healthy collage age individuals who are not professional athletes.

BMI is commonly used to classify individuals into different health risk categories. However, using BMI as an indicator of body composition could be inaccurate and may lead to bias in evaluating health conditions. For example, a shorter and/or more muscular individual may have a larger BMI than others, but they are not necessarily more overweight or having a higher health risk than others. Results of the current study also indicate that BMI is poorly correlated with body fat percentage measured with skinfold, BIA, or DXA. In addition to BMI, clinicians should consider other health and fitness parameters to have an accurate and comprehensive evaluation.

High intraclass correlations of all three methods demonstrate high levels of test-retest consistency by the examiner and assessment tools. Because an experienced skinfold examiner and the expensive DXA machine may not be available in smaller clinics and fitness/heath facilities, it is encouraging that the inexpensive and commonly available BIA machine may be able to provide reliable measurements. However, operator errors and subject variables (e.g., hydration status, skin condition) can still occur and not all BIA machines were made of the same quality. More importantly, the current study shows that BIA measurement significantly underestimated body fat percentage when compared to DXA measurement. With the BIA to DXA prediction equation, clinicians can provide subjects a more accurate body fat estimation and better evaluate health risks for those individuals. 
Citation: Grove J, Hung YJ. Body fat prediction equations for skinfold and bioelectrical impedance analysis using dual-energy x-ray absorptiometry data as the criterion. J Phys Ther Sports Med 2017;1(1):5-11.

Body fat percentage measured with DXA was significantly higher than those measured with skinfold and BIA. However, data from all three testing were highly correlated and DXA prediction equations were generated for skinfold and BIA data. When constructing the prediction equation for skinfold measure, it was noted that the data from DXA is slightly better correlated with the sum of the three skinfolds than with the final body fat percentage measured by the skinfold technique. This finding is consistent with Ball and Swan (2004) who also found a better correlation between DXA and skinfold using the sum of the 3-site measures [3,27]. Without going through 2 sets of conversion (from skinfold to body density and from body density to body fat percentage), there is a lesser chance for errors to occur during the process. In addition, it is also more convenient for clinicians in a practical setting.

For the current study, body fat percentage measured with DXA was averaged $21.35 \%$ for males and $30.19 \%$ for females. According to ACSM fitness categories for individuals 20-29 years old [10], male subjects of the current study would be categorized as "poor" and female subjects would be categorized as between "Poor" and "Very Poor". However, most of the subjects were very active and fit college students. Because body fat percentage measured with DXA is often higher than other measurements, this study is in agreement with $[3,27]$ that there is a need to re-examine the normative values of body fat percentage using newer technique (such as DXA), and update the literature and recommendation accordingly $[3,27]$.

This research is part of the fulfilment for Dr. Jared Grove's doctoral work at Angelo State University. The preliminary version of the manuscript was stored at Angelo State Digital Repository. This work has not been published by other journals and is not being submitted for publication elsewhere.

\section{Conclusion}

Skinfold and BIA measurements significantly underestimate body fat percentage compared to DXA measurements for body composition in college age subjects. It was suggested that DXA is a valid and reliable tool for body composition analysis. When it is unable to obtain a DXA measurement, adjustment equations for skinfold and BIA should be utilized to predict body fat percentage analysed by DXA.

\section{Limitations}

The majority of the subjects were very active (56\%) and young (18-27 years old) Caucasians (87\%). Therefore, the result of the current study may not be applicable for those individuals with a different activity level, age, or ethnic origin. Further research is needed to investigate DXA prediction equations over other age ranges, activity level, and ethnic populations. In addition, subjects were not excluded if they had systemic conditions and/or taking heart medication, which may have an impact on the results. Moreover, $75 \%-25 \%$ subject crossvalidation for those gender specific regression equations could not be performed due to the small sample size. Future studies with a larger sample size with multi-testing sites may be beneficial.

\section{Acknowledgement}

We appreciate Dr. Patricia Hutchinson's assistance in the statistically analysis of this manuscript.

\section{References}

1. Ogden CL, Carroll MD, Kit BK, et al. Prevalence of childhood and adult obesity in the United States, 2011-2012. JAMA. 2014;311:806-14.

2. Rothman KJ. BMI-related errors in the measurement of obesity. Int J Obes (2005). 2008;32: (S3)56-9.

3. Ball SD, Swan PD, Desimone R. Comparison of anthropometry to dual energy X-ray absorptiometry: a new prediction equation for women.Res Q Exerc Sport. 2004;75(3):248-58.

4. Clasey JL, Kanaley JA, Wideman L, et al. Validity of methods of body composition assessment in young and older men and women. J Appl Physiol (1985). 1999;86(5): 1728-38.

5. Heymsfield SB, Wang J, Kehayias J, et al. Chemical determination of human body density in vivo: relevance to hydrodensitometry.. Am j Clin Nutr. 1989;50 (6):1282-9.

6. Withers RT, Laforgia J, Pillans RK, et al. Comparisons of two-, three-, and four-compartment models of body composition analysis in men and women. J Appl Physiol (1985). 1998;85(1):238-45.

7. Davies JO, Davies ER, Howe K, et al. Practical applications of a monoclonal antibody (NDOG2) against placental alkaline phosphatase in ovarian cancer.J R Soc Med. 1985;78(11):899-905.

8. Jackson AS, Pollock ML, Ward A. Generalized equations for predicting body density of women. Med Sci Sports Exerc. 1980;12(3):175-81.

9. Jackson AS, Pollock ML. Generalized equations for predicting body density of men. Br J Nutr. 1978;40(3): 497-504.

10. Pescatello LS. ACSM's guidelines for exercise testing and prescription 9th ed. 2014. J Can Chiropr Assoc. 2014;58(3): 328.

11. Dehghan M, Merchant AT. Is bioelectrical impedance accurate for use in large epidemiological studies? Nutr J. 2008;7:26.

12. Hendel HW, Gotfredsen A, Hojgaard L, et al. Change in fat-free mass assessed by bioelectrical impedance, total body potassium and dual energy X-ray absorptiometry during prolonged weight loss. Scand J Clin Lab Invest. 1996;56(8):671-9.

13. Duren DL, Sherwood RJ, Czerwinski SA, et al. Body composition methods: comparisons and interpretation. J Diabetes Sci Technol. 2008;2(6):1139-46.

14. Tanner SB. Dual-energy X-ray absorptiometry in clinical practice: new guidelines and concerns. Current opinion in rheumatology. 2011;23:385-8. 
15. Bilsborough JC, Greenway K, Opa D, et al. The accuracy and precision of DXA for assessing body composition in team sport athletes. J Sports Sci. 2014;32(9):1821-8.

16. Chen W, Wilson JL, Khaksari M, et al. Abdominal fat analyzed by DEXA scan reflects visceral body fat and improves the phenotype description and the assessment of metabolic risk in mice.Am J Physiol Endocrinol Metab. 2012;303(5):635-43.

17. Ellis KJ. Human body composition: in vivo methods. Physiol Rev. 2000;80(2):649-80.

18. Gerbaix M, Metz L, Ringot E, et al. Visceral fat mass determination in rodent: validation of dual-energy X-ray absorptiometry and anthropometric techniques in fat and lean rats. Lipids Health Dis. 2010;9:140.

19. Kullberg J, Brandberg J, Angelhed JE, et al. Whole-body adipose tissue analysis: comparison of MRI, CT and dual energy X-ray absorptiometry. Br J Radiol. 2009;82(974): 123-30.

20. Senn SM, Kantor S, Leury BL, et al. In vivo quantification of fat content in mice using the Hologic QDR 4500A densitometer. Obes Res clin Prac. 2007;1:1-78.

21. Swennen Q, Janssens GPL, Geers R, et al. Validation of dual-energy $\mathrm{x}$-ray absorptiometry for determining in vivo body composition of chickens. Poult Sci. 2004;83(8): 1348-57.

22. Taylor AE, Kuper H, Varma RD, et al. Validation of dual energy X-ray absorptiometry measures of abdominal fat by comparison with magnetic resonance imaging in an Indian population. PLoS One. 2012;7(12):51042.

23. Bridge P, Pocock NA, Nguyen $T$, et al. Validation of longitudinal DXA changes in body composition from preto mid-adolescence using MRI as reference. J Clin Densitom. 2011;14:340-7.

24. Wagner DR, Heyward VH. Techniques of body composition assessment: a review of laboratory and field methods. Res Q Exerc Sport. 1999;70(2):135-49.

25. Chen Z, Wang Z, Lohman $T$, et al. Dual-energy X-ray absorptiometry is a valid tool for assessing skeletal muscle mass in older women. J Nutr. 2007;137(12):2775-80.

26. Fields DA, Teague AM, Short KR, et al. Evaluation of DXA vs. MRI for body composition measures in 1-month olds. Pediatr obes. 2015;10(5):8-10.

27. Ball SD, Altena TS, Swan PD. Comparison of anthropometry to DXA: a new prediction equation for men. Eur J Clin Nutr. 2004;58(11):1525-31.

28. Kuriyan R, Thomas T, Ashok S, et al. A 4-compartment model based validation of air displacement plethysmography, dual energy X-ray absorptiometry, skinfold technique \& bio-electrical impedance for measuring body fat in Indian adults. Indian J Med Res. 2014;139 (5):700-7.
29. Lohman TG, Harris M, Teixeira PJ, et al. Assessing body composition and changes in body composition. Another look at dual-energy X-ray absorptiometry. Ann N Y Aca Sci. 2000; 904:45-54.

30. Snijder MB, Visser M, Dekker JM, et al. The prediction of visceral fat by dual-energy X-ray absorptiometry in the elderly: a comparison with computed tomography and anthropometry. International journal of obesity and related metabolic disorders. Int $\mathrm{J}$ Obes Relat Metab Disord. 2002;26(6):984-93.

31. Pietrobelli A, Formica C, Wang Z, et al. Dual-energy X-ray absorptiometry body composition model: review of physical concepts.Am J Physiol. 1996;271(6 pt 1):E941-51.

32. Lang P, Steiger P, Faulkner K, et al. Osteoporosis. Current techniques and recent developments in quantitative bone densitometry. Radiol Clin North Am. 1991;29:49-76.

33. Santos DA, Gobbo LA, Matias CN, et al. Body composition in taller individuals using DXA: A validation study for athletic and non-athletic populations.J Sports Sci. 2013;31(4):405-13.

34. Silva AM, Heymsfield SB, Sardinha LB. Assessing body composition in taller or broader individuals using dualenergy X-ray absorptiometry: a systematic review. European J Clin Nutr. 2013;67:1012-21.

35. Clasey JL, Bradley KD, Bradley JW, et al. A new BIA equation estimating the body composition of young children. Obes Silver Spring, Md. 2011;19(9):1813-7.

36. Guedes AD, Bianco B, Lipay MVN, et al. A specific bioelectrical impedance equation to predict body composition in Turner's syndrome. Arq Bras Endocrinol Metabol. 2010;54(1):24-9.

37. Trippo U, Koebnick C, Zunft HJF, et al. Bioelectrical impedance analysis for predicting body composition: what about the external validity of new regression equations? Am J Clin Nutr. 2004;79:335-6; author reply 336-7.

38. Verovska R, Lacnak Z, Haluzikova D, et al. [Comparison of various methods of body fat analysis in overweight and obese women]. Int Med. 2009;55(5):455-61.

39. Bland JM, Altman DG. Statistical methods for assessing agreement between two methods of clinical measurement. Lancet. 1986;1(8476):307-10.

*Correspondence to:

You-jou Hung

Department of Physical Therapy

Angelo State University

USA

E-mail: you-jou.hung@angelo.edu 